\title{
Making the Case for Health Literacy
}

\author{
Sheryl Ness
}

Published online: 2 July 2012

(C) Springer Science+Business Media, LLC 2012

The key to a patient's understanding of the complexities of their cancer care experience is patient-provider communication. Healthcare providers and educators are recognizing that quite often, there is a substantial disconnect in knowledge and understanding between what is communicated and what is actually understood by the patient. Having cancer is complicated; understanding all of the nuances of their care, starting with the diagnosis, followed by treatment and then follow-up care is a bit like learning a new language. Add to this, the shock, fear, and other emotions experienced by a patient when hearing the word "cancer" makes it even more difficult to comprehend complex information.

For many years, educators have been focused on making the patient education experience a quality one. Initiatives have focused on conducting a thorough needs assessment, taking into consideration the person's baseline knowledge, learning preferences, culture, literacy level, and language. However, we may have been missing a key element to real understanding - health literacy.

Health literacy is defined as the degree to which individuals have the capacity to obtain, process, and understand basic health information and services needed to make appropriate health decisions [1]. Research shows that around $40 \%$ of all adults lack the level of health literacy needed to manage their own health. In addition, and directly relevant to cancer care, research reports that $66 \%$ of people age 60 and older have inadequate or marginal health literacy skills [2]. Limited health literacy impacts a person's ability to find and understand health information, manage chronic disease, and make informed decisions. For healthcare providers and educators, health literacy is a key factor when working with patients of any age, culture, education, and socioeconomic status [3].

S. Ness $(\bowtie)$

Mayo Clinic College of Medicine,

Rochester, MN 55905, USA

e-mail: ness.sheryl@mayo.edu
In 2010, the US Department of Health and Human Services published the National Action Plan to Improve Health Literacy. One of the primary goals of this plan is to "Promote changes in the health care system that improve health information, communication, informed decision making, and access to health services [4]." This plan serves as a road map to work together to bridge the gap between patients and providers and improve health literacy. Healthcare organizations are making progress, but the road ahead continues to have opportunities for improvement.

With all of this in mind, the Cancer Patient Education Network (CPEN) formed a task force in early 2011 to address the issue of health literacy within the practice of cancer education. CPEN also recognized that educators need to have access to resources and tools to assist in addressing health literacy in their practice. Task force members from around the globe joined forces to create a health literacy toolkit with interventions and strategies that had been studied and proven to improve health literacy. The toolkit includes resources that address healthcare provider awareness and education, patient self-management and strategies for alternative document design and delivery. The toolkit is posted on the CPEN website http://www.cancerpatienteducation.org/ health-literacy.shtml\#I and available for use for educators and healthcare providers.

One of the primary strategies that are making a positive impact on health literacy is the plain language initiative. Plain language is a global movement with a strong research base and backed by international leaders and organizations. In 2010, President Barack Obama also signed the Plain Writing Act of 2010 which requires the federal government agencies to write all public documents with elements of plain language.

Plain language is clear, concise, and easy to read. It is understood the first time you read it and in the shortest time possible. The priority is the reader, and includes best practices of proper grammar, organization, and word usage that 
helps the reader understand complex abstract concepts. Plain language is organized in a way that is logical and easy to read and follow. Broad concepts are introduced first, followed by detailed information and action points for the reader. The design elements of plain language incorporate visual space, cues, images, and breaks to help the reader navigate through the text and remember key points [5].

For people diagnosed with cancer, health literacy is a huge challenge. Cancer is complicated. Here is an example of a sentence that a patient might hear during a visit with their oncologist... "You have adenocarcinoma of the colon. It has metastasized to the liver, and may be causing your fatigue and low hemoglobin. We would like to treat you with a novel biological therapy that has been found to be effective for this type of carcinoma." With plain language in mind, the explanation could have gone like this - "You have cancer of the large intestine. It has spread to the liver, and may be making you feel tired and to have a low red blood cell count. We would like to treat you with a new drug that works well for this type of cancer."

Even as healthcare professionals, we can probably all think of a time when a conversation with our physician went over our head. Using plain language in our healthcare education and communication can make a vital difference. Keep this in mind the next time you are writing a new education piece or explaining a complicated diagnosis or treatment to a patient.
Health literacy will be a focus at the upcoming International Cancer Education Conference in Ann Arbor, Michigan from September 19-22, 2012. This meeting is collaboratively hosted by the American Association for Cancer Education (AACE), the Cancer Patient Education Network (CPEN), and the European Association for Cancer Education (EACE). For more information on this meeting, see http://cme.ucsd.edu/canceredu2012/.

\section{References}

1. US Department of Health and Human Services (2000) Healthy People 2010 (2nd ed.) [with Understanding and Improving Health (vol. 1) and Objectives for Improving Health (vol. 2)]. US Government Printing Office: Washington, DC

2. Doak CC, Doak LG, Root JH (1996) The literacy problem. In: Teaching patients with low literacy skills, 2nd ed. Lippincott: Philadelphia. pp 1-9

3. Berkman ND, DeWalt DA, Pignone MP, Sheridan SL, Lohr KN, Lux L., et al. (2004) Literacy and health outcomes (AHRQ publication no. 04-E007-2). Agency for Healthcare Research and Quality: Rockville, MD

4. US Department of Health and Human Services, Office of Disease Prevention and Health Promotion (2010) National action plan to improve health literacy. US Department of Health and Human Services: Washington, DC

5. US Department of Health and Human Services, National Institutes of Health (NIH). An introduction to plain language at NIH. http:// www.nih.gov/clearcommunication/plainlanguage.htm. Accessed: 21 May 2012 